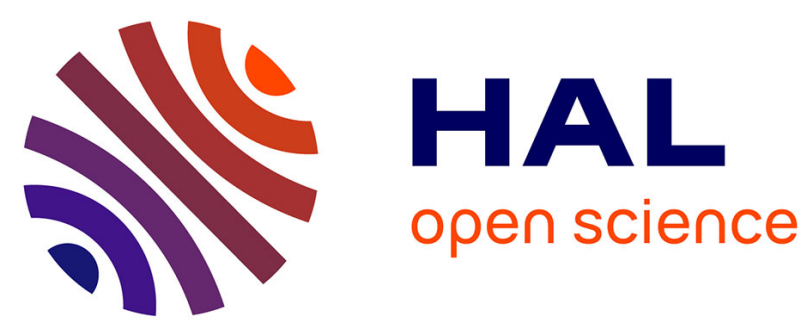

\title{
An atomistic study on the activation enthalpies for interface mobility and boundary diffusion in an interface-controlled phase transformation
}

\author{
Cornelis Bos, Ferdinand Sommer, Eric Jan Mittemeijer
}

\section{To cite this version:}

Cornelis Bos, Ferdinand Sommer, Eric Jan Mittemeijer. An atomistic study on the activation enthalpies for interface mobility and boundary diffusion in an interface-controlled phase transformation. Philosophical Magazine, 2008, 87 (16), pp.2245-2262. 10.1080/14786430601156201 . hal-00513813

\section{HAL Id: hal-00513813 \\ https://hal.science/hal-00513813}

Submitted on 1 Sep 2010

HAL is a multi-disciplinary open access archive for the deposit and dissemination of scientific research documents, whether they are published or not. The documents may come from teaching and research institutions in France or abroad, or from public or private research centers.
L'archive ouverte pluridisciplinaire HAL, est destinée au dépôt et à la diffusion de documents scientifiques de niveau recherche, publiés ou non, émanant des établissements d'enseignement et de recherche français ou étrangers, des laboratoires publics ou privés. 


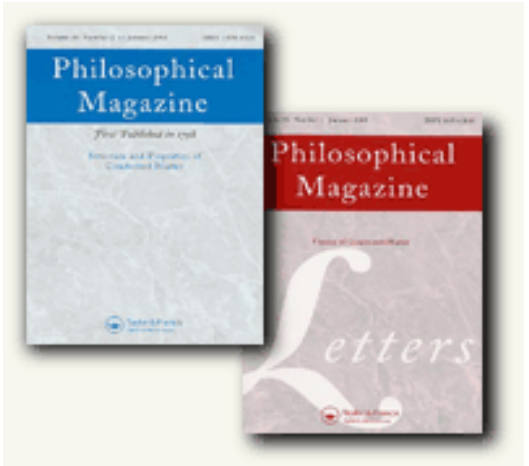

\section{An atomistic study on the activation enthalpies for interface mobility and boundary diffusion in an interface-controlled phase transformation}

\begin{tabular}{|c|c|}
\hline Journal: & Philosophical Magazine \& Philosophical Magazine Letters \\
\hline Manuscript ID: & TPHM-06-Jun-0232.R1 \\
\hline Journal Selection: & Philosophical Magazine \\
\hline $\begin{array}{r}\text { Date Submitted by the } \\
\text { Author: }\end{array}$ & $10-N o v-2006$ \\
\hline Complete List of Authors: & $\begin{array}{l}\text { Bos, Cornelis; Max Planck Institute for Metals Research } \\
\text { Sommer, Ferdinand; Max Planck Institute for Metals Research } \\
\text { Mittemeijer, Eric Jan; Max Planck Institute for Metals Research }\end{array}$ \\
\hline Keywords: & grain boundary diffusion, Monte-Carlo, phase transformations \\
\hline Keywords (user supplied): & interface migration, iron \\
\hline
\end{tabular}

\section{S ScholaroNE \\ Manuscript Central}




\title{
An atomistic study on the activation enthalpies for interface mobility and boundary diffusion in an interface-controlled phase transformation
}

\author{
C. BOS, F. SOMMER AND E. J. MITTEMEIJER* \\ Max Planck Institute for Metals Research, Heisenbergstr. 3, \\ D-70569, Stuttgart, Germany
}

\begin{abstract}
A multi-lattice kinetic Monte Carlo atomistic simulation method has been used to simulate the austenite to ferrite interface-controlled transformation in pure iron. By performing simulations with different amounts of "free volume" at the interface quantative relations between the activation energies for interface mobility, boundary self diffusion and bulk self diffusion have been investigated. Furthermore, the effect of different interface orientations on the distribution of the free volume over the interface, as determined by the interface crystallography, and its consequences for the interface mobility activation energy has been evaluated. The essential role of thermal fluctuations in the free volume distribution at the interface has been shown. The activation enthalpy for boundary diffusion is shown to be larger than the interface mobility activation enthalpy.
\end{abstract}

\begin{abstract}
Keywords: phase transformation; Monte Carlo techniques; grain boundary diffusion; interface migration; iron
\end{abstract}

\section{Introduction; discussion of current state of knowledge}

In interface controlled solid-state transformations, processes at the interface determine the transformation rate. Typical examples of interface controlled transformations are massive transformations and grain growth. In a massive transformation one phase is transformed into another phase, as for example in the massive austenite $(\gamma)$ to ferrite $(\alpha)$ transformation which can be observed in many iron-based alloys. In grain growth the product and parent phases are the same but the two crystals are differently oriented. For grain growth the driving force for the transformation will be generally much lower than for interface controlled phase transformations. The text-book equation for the interface velocity, $v$, reads [1]

*email to: e.j.mittemeijer@mf.mpg.de 


$$
v(T)=M\left(1-\exp \left(-\frac{\Delta G}{k_{B} T}\right)\right),
$$

where $k_{B}$ is the Boltzmann constant, $\Delta G$ is the driving force per atom and with the socalled mobility $M$ according to

$$
M=M_{0} \exp \left(-\frac{\Delta H^{a}}{k_{B} T}\right),
$$

where $M_{0}$ is a pre-exponential constant, $\Delta H^{a}$ is the activation enthalpy for the interface mobility and $T$ is the temperature (the entropy contribution has been included in the pre-exponential constant).

The theoretical background of equation (1) is illustrated for the $\gamma$ to $\alpha$ phase transformation in figure 1 . The interface is assumed to move by independent thermally activated jumps 'across' the interface. Because atoms crossing the interface experience a higher total energy barrier in the direction from $\alpha$ to $\gamma$ than from $\gamma$ to $\alpha$, more atoms will jump from $\gamma$ to $\alpha$ than from $\alpha$ to $\gamma$, leading to growth of the $\alpha$ phase. The difference in the total energy barriers of the two process directions is equal to $\Delta G$ (in figure 1: $\Delta G_{\gamma, \alpha}$ ). This simple model for the interface velocity usually can well describe experimental observations, especially if the temperature range considered is not too large.

\section{[Insert figure 1 about here]}

Within the context of this simple picture, it can be argued [1] that the energy of activation for the migration of atoms within the $\alpha$ phase probably is an upper bound for the magnitude of $\Delta H^{a}$ and furthermore, if the boundary is incoherent, $\Delta H^{a}$ can be expected to approximate the activation energy for grain boundary diffusion, $\Delta H_{G B}^{D}$. In other words: the mobility of the atoms at the boundary determines the magnitude of $\Delta H^{a}$. However the picture as sketched above is much too simple in general, as shown recently (see the discussion below at equations (6) and (7) and the discussion in Ref. $[2])$.

The mobility of atoms can be described by the jump rate of the atoms. In simple transition state theory (STST, see for example Ref. [3]) the atomic jump rate, $k$, is given by

$$
k=v_{0} \exp \left(-\frac{E^{a}}{k_{B} T}\right)
$$

where $v_{0}$ is a frequency factor and $E^{a}$ is the jump activation energy. If $\Delta U$ denotes the change in system energy caused by the atomic jump considered ${ }^{\dagger}$, then for energetically unfavourable jumps (i.e. $\Delta U>0$ ) $E^{a}$ can be written as (see figure 2)

$$
E^{a}=\Delta U+Q
$$

\footnotetext{
${ }^{\dagger}$ All energies associated with atomic jumps are denoted by $U$ in this text.
} 
For energetically favourable jumps (i.e. $\Delta U<0) E^{a}$ obeys (see figure 2):

$$
E^{a}=Q
$$

[Insert figure 2 about here]

Figures 1 and 2 look similar and thereby it is suggested that $\Delta H^{a}$ can be identified as $Q$ of a single atomic jump at the boundary. This impression is false. An atom can only jump to a volume of free space large enough to accommodate it (e.g. a vacancy in a crystal lattice). This means that in $\Delta H^{a}$ the enthalpy of formation of such a free volume must be included. $E^{a}$ (=Q for a favourable jump) represents (only) the activation energy for migration. It has been shown that the formation of a suitable free volume requires jumps of a number of different atoms.

Recently, atomistic multi-lattice kinetic Monte Carlo (ml-kMC) simulations [4] of lateral growth of $\alpha$ in a $\gamma$ to $\alpha$ phase transformation in iron have shown that series of jumps of groups of atoms determine $\Delta H^{a}$ (the groups of atoms do not jump simultaneously but one after another) [2,5]. It is important to note that this atomistic multi-lattice kMC simulation method has been based on the same concept as the one behind equation (1): the interface moves by thermally activated jumps (with the jump rate given by equation (3)), with the exception that now all jumps at the interface are considered and not only jumps across the interface. Hence, figure 1 has to be replaced by figure 3 which shows that multiple jumps contribute to $\Delta H^{a}$ and that multiple intermediate states exist on the energy path from $\gamma$ to $\alpha[2,5]$.

\section{[Insert figure 3 about here]}

The series of (energetically unfavourable) jumps by groups of atoms are required to create a path from $\gamma$ to $\alpha$. This is illustrated in figure 4: $\gamma$ atoms at the $\gamma / \alpha$ interface cannot jump to the empty $\alpha$ sites because neighbouring $\gamma$ atoms are blocking those sites. Only with a rearrangement of the free space available at the interface can these $\gamma$ atoms jump to $\alpha$ lattice sites. Consequently $\Delta H^{a}$ must contain a formation enthalpy of a free volume.

[Insert figure 4 about here]

As discussed above, $\Delta H_{G B}^{D}$ should be a good estimate for $\Delta H^{a}$ for incoherent boundaries. However, experimental results, that can be summarized as [6]

$$
\frac{\Delta H_{G B}^{D}}{\Delta H_{V}^{D}} \in\left[\frac{1}{2}, \frac{2}{3}\right]
$$

and

$$
\frac{\Delta H^{a}}{\Delta H_{V}^{D}} \geq \frac{2}{3}
$$


with $\Delta H_{V}^{D}$ the activation enthalpy for volume (bulk) diffusion, show that $\Delta H^{a}$ can be considerably larger than $\Delta H_{G B}^{D}$. Mott [7] has already suggested that groups of atoms may be activated instead of single atoms, which could explain the difference of $\Delta H^{a}$ and $\Delta H_{G B}^{D}$. However, this difference has been usually attributed to the effect of impurities or inclusions present at the boundary [1]. Support for the idea that multiple jumps contribute to $\Delta H^{a}$ for interface-controlled phase transformations [2,5] can also be derived from molecular dynamics (MD) simulations of grain growth [8-10]. Strikingly, however, in contrast with the experimental results described by equations (6) and (7), the values found for $\Delta H^{a}$ in the MD simulations are lower than $\Delta H_{G B}^{D}$. This discrepancy has been attributed to impurity effects that always occur in real experiments but not in the simulations [8,9].

In this work the activation enthalpies of interface mobility, boundary self diffusion and bulk self diffusion have been determined and compared to see if a theoretical understanding can be given for the experimental expectations expressed by equations (6) and (7). To this end, jump specific activation energies $E^{a}$ have been considered, recognizing that for jumps at the interface $E^{a}$ depends on the exact local surroundings of the jumping atom. Lateral growth of the $\alpha$ (bcc) product phase in a $\gamma$ (fcc) to $\alpha$ (bcc) transformation in a single element system (iron) has been simulated. Different interface orientations have been used in the simulation, but always with the closest packed planes parallel at the interface (i.e. fcc(111)//bcc(110)). Although here planar growth is simulated in a 3-dimensional simulation setup, the results should apply to full 3-dimensional growth as well, as the structure at the interface line of the 2dimensional bcc seed (see also figure 5, plane B) is incoherent. The choice to simulate lateral growth was made for computational efficiency reasons. The simulation method and simulation settings are described in section 2. The results are presented in section 3. The discussion in section 4 focuses on the activation enthalpies for interface mobility and boundary self diffusion.

\section{Simulation method and settings}

The multi-lattice kMC method as described in Refs. [2,4,5] allows the simulation of a phase transformation from one crystal structure to another, by incorporating both crystal structures as possible sites for the atoms. To allow the atoms at and near the moving interface to take intermediate positions between the lattice sites, a collection of randomly placed sites can also be included. These intermediate positions allow for irregularities in the atomic structure of transformation interfaces[4].

A transformation is simulated by letting the atoms at the interface jump to a neighbouring empty site. The jump rate constant, $k$, is calculated with equation (3). The jump activation energy, $E^{a}$, is calculated using a constrained conjugate gradient energy minimization method as presented in Ref. [11]. To reduce the calculation time of the simulations a neural network has to be trained with data from over ten thousand jumps. The trained neural network can then be used in the simulations to calculate $E^{a}$ (with a mean square error of less than $2 \%$ ).

In all simulations a value of $10^{13}\left(\mathrm{sec}^{-1}\right)$ has been used for $v_{0}$ (cf. equation (3)). The atomic interaction energies have been calculated using the Johnson-Oh embedded atom method potential for iron [12]. 


\section{$2.1 \quad$ Interface orientations}

The start configuration involves a bcc seed for the simulation of lateral growth in atomic plane $\mathrm{B}$ for the structure shown in figure 5 . The interface is always parallel to both an fcc (111) plane and a bcc (110) plane, with the distance between planes B and $\mathrm{C}$ (from figure 5) equal to the equilibrium bcc (110) lattice spacing and with the distance between planes A and B equal to the equilibrium fcc (111) lattice spacing. Four different interface systems have been created for the simulations by rotating the bcc crystal around the axis perpendicular to the interface over an angle $\theta$, leading to the four orientations shown in table 1.

\section{[Insert figure 5 about here]}

\section{[Insert Table 1 about here]}

The (2-dimensional) bcc seed (in plane B, cf. figure 5) has been generated by first defining two lines (in plane B) and removing all fcc atoms and filling all bcc sites in between these lines. Then every empty lattice site (in the interface region) that is surrounded by a large enough pocket of free space is filled with an atom if the addition of the atom lowers the total system energy (first bcc sites are filled then fcc). Because of the orientation specific misfit at the interface lines this leads to a different number of total atoms in the system for the systems A to D (more fcc atoms are taken out than bcc atoms can be put in). A different number of total atoms in the system leads to a different amount of free volume at the interface. To create the same amount of free volume per interface line length (of the 2-dimensional bcc seed) for all systems, extra vacancies have been introduced to systems A, C and D. Vacancies are created by removing fcc atoms close to the interface lines in plane B in figure 5 . The exact position of the removed atoms is not important because vacancies are strongly bound to the interface. System A has also been used in simulations without any added vacancies (see section 3.1), to avoid confusion this will be called system $\mathrm{A}_{2}$.

The fcc and bcc crystals have been created with equal planar density for the planes parallel to the interface (with bcc number density of $84.9 \cdot 10^{27}$ atoms $/ \mathrm{m}^{3}$ ). Periodic boundaries have been used in both the $x$ and $y$ directions (see figure 5). The random sites have been distributed according to a restrictedly random distribution: First, the system is divided in small cubic cells with a side length of $1.4 \cdot r_{b}$ (with $r_{b}$ the bcc nearest neighbour distance). Then, $N_{\text {ran }}$ random sites are inserted into each cell according to a uniform distribution. With a higher value for $N_{\text {ran }}$ the jumping atoms can take more different positions. In reality any position can be considered as a potential atom site. Therefore with a higher value for $N_{\text {ran }}$ a closer approximation of reality is obtained. However, if many random sites are used then there will be many random sites very close to lattice positions (or other random sites). If sites are very close to each other, then, from an energy point of view they will be practically indistinguishable. This means that the activation energy for jumps between sites that are very close to each other will be very low and therefore these jumps occur very often (see Ref. [2]). If this happens often (i.e. if $N_{\text {ran }}$ is high) then almost all calculation time will be used for these very short jumps (that do not contribute to the transformation). This renders transformation simulations with very high values for $N_{\text {ran }}$ useless. 
The driving force for the transformation is determined by the iron EAM potential and, for the densities of the fcc and bcc crystals used here (see above), it is $\Delta U_{b c c, f c c}=$ $0.03 \mathrm{eV}$ (= fcc-bcc energy difference). With this driving force and the chosen interface orientations the chance of bcc nucleating in plane A (cf. figure 5) is extremely small (cf. Ref. [4]) and therefore only lateral growth in plane B occurs. Because the positions of the lattice sites are fixed, temperature has no influence on the geometry of the interface through density changes. The only influence temperature has is on the atomic jump rate. For optimal calculation time efficiency (see Appendix $\mathrm{B}$ of Ref. [2]) the simulations have been performed in a temperature range from 2000 to $3000 \mathrm{~K}$.

\subsection{Interface Mobility}

The interface mobility activation enthalpy (here denoted as $\Delta H^{a, s}$, i.e. with an additional superscript 's'; cf. equation (2), to signify that it is the activation enthalpy as determined from the simulations) has been determined by a non-linear least squares fit to the obtained data for the transformation rate, $r_{\mathrm{tf}}{ }^{\ddagger}$, according to:

$$
r_{\mathrm{tf}}(T)=C \exp \left(-\frac{\Delta \mathrm{H}^{a, s}}{k_{B} T}\right)\left(1-\exp \left(-\frac{\Delta U_{b c c, f c c}}{k_{B} T}\right)\right)
$$

where $C$ is a pre-exponential constant and $\Delta U_{b c c, f c c}$ is the fcc-bcc energy difference $(=0.03 \mathrm{eV})$.

\subsection{Boundary diffusion}

The activation enthalpy for boundary self diffusion $\left(\Delta H_{G B}^{D}\right)$ can be written as the sum of the vacancy formation enthalpy, $\Delta H_{G B}^{f}$, and the activation enthalpy for vacancy migration, $\Delta H_{G B}^{m}$, (both at the boundary),

$$
\Delta H_{G B}^{D}=\Delta H_{G B}^{f}+\Delta H_{G B}^{m}
$$

The activation enthalpy for vacancy migration in boundary self diffusion, $\Delta H_{G B}^{m}$, has been obtained from mean square atom displacements (MSD) measurements [13] in a system with a completely flat interface (i.e. no bcc seed in plane B of figure 5) with a single vacancy at the interface (i.e. in plane B of figure 5). With a flat interface no vacancies are formed during the simulations, which means that the displacements of the atoms are all effectuated by the movement of the single vacancy. The vacancy is free to move away from the interface but seldomly does so because a vacancy jump away from the interface is energetically highly unfavourable.

The summed square atom displacement (SSD) is given by

\footnotetext{
${ }^{\ddagger}$ An estimate for the transformation rate is determined by measuring the time between $20 \%$ and $80 \%$ completion of the transformation of the remaining fcc atoms in plane B as shown in figure 5 . In equation (8) the entropy contribution to the driving force is neglected (cf. equation (1)).
} 
where $t$ is time, $N_{G B}$ is the number of atoms at the boundary (atoms with neighbours of both fcc and bcc type) and $\boldsymbol{r}_{j}$ is the position of atom $j$. If $a$ represents the slope of a SSD vs. time plot, then $\Delta H_{G B}^{m}$ can be found by a fit of

$$
\ln (a)=C^{\prime}-\frac{\Delta H_{G B}^{m}}{k_{B} T},
$$

with $C^{\prime}$ as a constant, to the data for $a$ obtained at several temperatures.

As noted in section 2.1, when a bcc seed is created more fcc atoms are removed than bcc atoms are put in. This leads to a certain amount of free space at the interface (i.e. there is an excess volume associated with the interface). When no extra vacancies are introduced to the system, this free space is spread out over the interface (here: along the interface line) in such a way that no single pocket of free space is large enough and at the right position to accommodate an atom on a lattice site. However, (especially during the transformation) the distribution of the free space will fluctuate and temporarily vacancies can be formed [14]. These vacancies can also be mobile, which means that diffusion can take place during the transformation. The diffusion will be primarily along the interface line because the vacancies are energetically bound to the interface line (cf. above equation (10)). The activation enthalpy for this diffusion can be found by measuring the SSD during the transformation as above. The atoms are also displaced because of the transformation. To minimize the contribution of the movement of the atoms due to the transformation, only those atoms that have moved more than $2^{1 / 2}$ times the bcc nearest neighbour distance are counted in the evaluation of the SSD. The activation enthalpy for diffusion now contains contributions from both vacancy formation and vacancy migration. Therefore, equation (11) must be replaced by

$$
\ln (a)=C^{\prime \prime}-\frac{\Delta H_{G B}^{D}}{k_{B} T},
$$

with $C^{\prime \prime}$ as a constant.

\section{Results and evaluation}

\subsection{Interface mobility in system $A_{2}$}

The activation enthalpy for the interface mobility for system $\mathrm{A}_{2}$ (=system A without added vacancies) has been determined with three different settings. With the bcc nucleus created as described in section 2.1, with two extra added atoms and with four extra added atoms. The interface mobility activation enthalpy for system $\mathrm{A}_{2}$ is shown as a function of $N_{\text {ran }}$ (a measure for the random site density, see section 2.1) in figure 6. An increase in the number of random sites in the system gives the atoms a greater freedom of movement. This will make it easier for the atoms to find a path from fcc to bcc (see figure 4 and the discussion in section 1) leading to a lower activation 
enthalpy, as shown in figure 6. The effect is not strong and increasing $N_{\text {ran }}$ beyond 12 does not appear to affect $\Delta H^{a, s}$ anymore.

[Insert figure 6 about here]

When extra atoms are added to the system the free space at the interface will be reduced. The extra atoms cannot be inserted directly at the start of the simulation because there is no free space in the form of vacancies (see section 2.1). However, during the transformation vacancies can be formed by thermal fluctuations (see section 2.3) and when this occurs an atom can be inserted on the free site. Thus, in separate experiments, 2 and 4 atoms have been added during the very early stages of the transformation. Because the extra atoms reduce the free space at the interface, it becomes more difficult for the atoms to find a path from fcc to bcc. This is reflected in the increase observed for $\Delta H^{a, s}$ when extra atoms are added to the system, as shown in figure 6 (as an exception with $N_{\text {ran }}=8, \Delta H^{a, s}$ is lowest for the system with 2 added atoms, but the trend is clear $)^{\S}$.

\subsection{Boundary diffusion in system $A_{2}$}

The boundary self diffusion migration activation enthalpy, $\Delta H_{G B}^{m}$, determined from SSD measurements with a single vacancy at a flat interface (see section 2.3) is shown for system $\mathrm{A}_{2}$ (for this case identical to system A) as a function of $N_{\text {ran }}$ in figure 7. It follows that $\Delta H_{G B}^{m}$ only changes slightly with $N_{\text {ran. }}$. For $N_{\text {ran }}=15$ no reasonable fit of equation (11) to the simulation data could be obtained. The bulk self diffusion migration activation enthalpy for the used simulation settings is

$$
\Delta H_{V}^{m}=1.34 \mathrm{eV}
$$

This value is, at first sight surprisingly, somewhat lower than the values obtained for $\Delta H_{G B}^{m}$ (cf. figure 7), which can be explained follows. For vacancy jumps in the bulk the energy change caused by the jump is always zero $(\Delta U=0)$ and therefore the activation energy for a bulk vacancy jump is always given by equation (5) and $\Delta H_{V}^{m}=Q_{V}$ (with $Q_{V}$ the $Q$-value for vacancy jumps in the bulk). At the interface, atoms at different positions will have different binding energies. This means that for vacancy jumps at the interface $\Delta U$ will generally not be zero. Vacancy jumps with a positive $\Delta U$-value are more difficult (cf. equations (4) and (5)) and will be rate limiting for the diffusion. For the case considered, apparently the $Q$-values for vacancy jumps at the interface are comparable in size to $Q_{V}$. That, on average, the $Q$ values for jumps at the interface are not considerably smaller than $Q_{V}$ is most likely caused by the chosen interface orientation involving two closest packed planes parallel at the interface. The combination of a contribution to $\Delta H_{G B}^{m}$ by a positive $\Delta U$

\footnotetext{
${ }^{\S}$ In the case of 4 added atoms the transformation was so difficult for $N_{\text {ran }}=0$ that no value for $\Delta H^{a, s}$ could be obtained. For high values of $N_{\text {ran }}$ most of the calculation time is spent on jumps of atoms between sites very close to each other that do not contribute to the transformation (see section 2.1). This made it impossible to obtain values for $\Delta H^{a, s}$ with $N_{\text {ran }}>=12$ with 4 added atoms within a reasonable simulation time (although a transformation does take place).
} 
and a relatively high value for $Q$ for jumps at the interface explains why $\Delta H_{G B}^{m}$ can be somewhat larger than $\Delta H_{V}^{m}$.

\section{[Insert figure 7 about here]}

The activation enthalpy for diffusion during the transformation ${ }^{* *}, \Delta H_{G B}^{D}$, has also been determined (cf. equation (12) in section 2.3). From the results shown in figure 7 it follows that $\Delta H_{G B}^{D}$ is approximately equal to $\Delta H_{G B}^{m}$ for system $\mathrm{A}_{2}$ with no extra added atoms. Because the start configuration does not contain any vacancies, $\Delta H_{G B}^{D}$ now does include a vacancy formation part (cf. section 2.3). Apparently, for the case considered, the vacancy formation enthalpy is approximately equal to zero, because $\Delta H_{G B}^{D} \approx \Delta H_{G B}^{m}$. This is ascribed to the relatively large amount of free space at the interface upon bcc nucleus creation, although care was taken to fill all possible lattice sites after creating the bcc nucleus (in plane B of figure 5). An intrinsically large amount of free space implies easy vacancy formation, as observed. If a part of the free space is taken out of the system it must become more difficult to form a vacancy. This is confirmed by simulations with extra added atoms as shown in figure 7: With two added atoms $\Delta H_{G B}^{D}$ is significantly larger than $\Delta H_{G B}^{m}$. However, when two more atoms are added (for a total of four) $\Delta H_{G B}^{D}$ drops again to $\Delta H_{G B}^{m \dagger \dagger}$. This may give rise to the impression that diffusion is faster with four added atoms (as compared to two added atoms). This impression is false, as the value for the constant $C^{\prime \prime}$ (cf. equation (12)) is much smaller for the system with four added atoms, as shown in table 2 for $N_{\text {ran }}=12$, leading to the smallest values for SSD in the temperature range considered here for the system with four added atoms (see also the discussion in section 4.2)

[Insert Table 2 about here]

\subsection{The interface mobility and its orientation dependence}

Values obtained for the activation enthalpy of the interface mobility, $\Delta H^{a, s}$ (and the corresponding pre-exponential constant), have been gathered in tables 3 and 4 for systems A to D with $N_{\text {ran }}=0$ and $N_{\text {ran }}=4$ respectively. Against expectation (with a view to the discussion on $\Delta H^{a, s}$ for system $\mathrm{A}_{2}$ in section 3.1) $\Delta H^{a, s}$ does not decrease slightly, if at all, but, in particular for system D, increases as $N_{\text {ran }}$ is increased from 0 to 4 . In section 3.1 it was argued that an increase in $N_{\text {ran }}$ increases the atomic mobility which should lead to a decrease of $\Delta H^{a, s}$. As discussed below, this effect is due to the specific misfit at the interface (of system D).

\footnotetext{
** The scatter in the SSD vs. time plots determined from transformation simulations is often very large. Even although the slope, $a$, of the SSD vs. time plots (cf. equation (12)) was averaged over at least ten simulation replicas, a reliable fit could not always be obtained. Therefore $\Delta H_{G B}^{D}$ could not be given for all values of $N_{\text {ran }}$ in figure 7.

${ }^{\dagger \dagger}$ Unfortunately, $\Delta H_{G B}^{D}$ could only be determined reliably for $N_{\text {ran }}=12$, but even although no good fit of equation (12) to the simulation data could be obtained with $N_{\text {ran }}=4$ and $N_{\text {ran }}=8$ those simulations still indicated similarly low values for $\Delta H_{G B}^{D}$.
} 


\section{[Insert Table 3 about here]}

[Insert table 4 about here]

In the ml-kMC framework (in the absence of random sites) the atoms are positioned on sites of either the product or parent lattice. Thus atoms at the interface must also take positions at ideal (bulk) lattice sites. This is a simplification of reality as it is well known that atoms at an interface can take intermediate, energetically more favourable positions, thereby relaxing the interface structure. The mismatch patterns for the interface orientations of systems A to D are shown in figure 8.

\section{[Insert figure 8 about here]}

The percentage of overlapping sites ${ }^{\text {t* }}$ (of the fcc and bcc lattices) is almost equal for these unrelaxed structures $(\sim 10 \%)$. When the interface is relaxed by a molecular statics energy minimization procedure (here the atoms are no longer fixed on the lattice sites), the overlap changes (i.e. increases) differently for the four different systems; see results in table 5. The (relaxed) interface energies, $\gamma$, have also been listed in table 5. Evidently, the interface energy, $\gamma$, is considerably lower for system D as compared to the other systems. A comparison of the results gathered in tables 3 and 4 with the data in table 5 reveals that $\Delta H^{a, s}$ increases significantly with decreasing $\gamma$. Random sites in the system give the atoms at the interface an opportunity to take intermediate positions between the lattice sites. Hence, introducing random sites can lead to some relaxation of the interface structure. The relaxation in system $\mathrm{D}$ is relatively strong, stabilizing the interface, and this can explain why $\Delta H^{a, s}$ increases when $N_{\text {ran }}$ increases for system D (although in general the random sites have a decreasing effect on $\left.\Delta H^{a, s}\right)$. The strong relaxation and the higher $\Delta H^{a, s}$ for system $\mathrm{D}$ can be ascribed to the specific mismatch situation for system D. The mismatch patterns presented in figure 8 show that in the unrelaxed system D large groups of neighbouring overlapping sites occur, which differs from the mismatch patterns for systems A, B and C. After relaxation for system D this will lead to local parts of the interface where the bcc and fcc lattices are almost coherent. In these parts of the boundary that are (nearly) coherent (see figure 9) it is very difficult for the atoms to find a path from fcc to bcc, because there is almost no free space available for movement of the atoms.

\section{General discussion}

\subsection{Interface mobility}

The results presented in sections 3.1 and 3.3 show that the random site density in the simulation system has two different, opposing effects on the interface mobility: (i) On the one hand an increase of the random site density increases the freedom of movement of the atoms at the interface making it easier for the atoms to find a path from fcc to bcc, leading to a lower value for the activation enthalpy for the interface

\footnotetext{
$\$$ Two sites are considered to overlap if the distance between the two sites is less than $15 \%$ of the bcc nearest neighbour distance.
} 
mobility (there will be less intermediate states in the reaction path and the $\Delta U$-values of the intermediate jumps are likely to be smaller (cf. figure 3)). (ii) On the other hand, an increase of the random site density also allows for enhanced relaxation of the interface which as a consequence can increase the interface mobility activation enthalpy. The net result of the two effects depends on the crystallography of the interface (see section 3.3).

The simulations with different amounts of free space at the transformation interface have shown that the interface mobility activation enthalpy increases with decreasing excess volume at the interface (section 3.1). The important role of excess volume associated with interfaces has also been found in studies on dynamic grain boundary behaviour. In a molecular dynamics study on grain boundary sliding in nano-crystalline material, it was found that atomic level shuffling and stress-assisted free-volume migration play an important role in the plastic deformation process occurring at the GB region [16], and, on the basis of a molecular dynamics study on a $\Sigma=5$ tilt GB in fcc material, it was found that excess volume is the key to both components of the migration mechanism (local volume fluctuations and atomic hops perpendicular to the boundary plane) [10].

The simulations with the different interface orientations have shown that not only the amount of excess volume but in particular also the distribution of the free volume along the interface is important (see section 3.3). Large groups of atoms associated with local, (nearly) coherent parts of the interface have an impeding effect on the interface mobility.

Any excess volume at a boundary is caused by the misfit of the atoms of the two different crystals at the boundary. The amount of such excess volume depends on the structure of the boundary. Highly disordered boundaries can be expected to have a large excess volume. But also symmetrical boundaries can have a considerable excess volume, as was shown by a high-resolution transmission electron microscopy study (HRTEM) of the $\Sigma=3$, (111) grain boundary in NiAl [17]. For stationary boundaries it is possible to determine the excess volume on the basis of HRTEM analysis. However, for moving boundaries it may be very difficult to get values for the excess volume as a function of the interface orientation.

In computer simulations the amount of excess volume present at the boundary is strongly influenced by the procedure used to construct the boundary in the start configuration of the simulation (unless the boundary is formed during a simulation and does not constitute a part of the initial configuration). It is common to first create a boundary with the atoms at the ideal lattice positions of the two crystals, where one atom of every pair atoms that come too close to each other is removed from the system, and then the interface is relaxed to a minimum energy [e.g. Refs. 8 and18]. In order to make comparison between simulations in different investigations possible it should be specified exactly how the interface has been generated.

The values obtained for the interface mobility activation enthalpy (see section 3.1) are lower than the bulk vacancy migration activation enthalpy (see section 3.2) (i.e. $\Delta H^{a, s}<\Delta H_{V}^{m}$ ). Therefore, the series of jumps that effectuate the transformation must have $Q$-values that are smaller than $\Delta H_{V}^{m}=Q_{V}$ (i.e. the $Q$-value of a bulk vacancy jump). This is in contrast to the $\Delta H_{G B}^{m}$-values of the vacancy jumps at the interface that effectuate boundary self diffusion (with $\Delta \mathrm{U}>0$; see section 3.2). The distribution of the free volume around the jumping atoms in transformation jumps is likely different from the distribution in vacancy jumps. Also with a view to the discussion in the preceding paragraph, it is remarked that the interface mobility activation enthalpy 
as obtained from the simulations, denoted by $\Delta H^{a, s}$, will be representative of the true interface mobility activation enthalpy, $\Delta H^{a}$, only if the amount of excess volume at the transformation interface is realistic.

\subsection{Boundary diffusion}

For the boundary diffusion the excess volume plays an equally important role as for the transformation interface mobility (see discussion in section 4.1). In section 3.2 it was shown that in system $\mathrm{A}_{2}$ with no added atoms, vacancy formation is very easy and $\Delta H_{G B}^{D} \approx \Delta H_{G B}^{m}$. This indicates that the excess volume at the interface was unrealistically large. When free space is removed from the interface by inserting two extra atoms, $\Delta H_{G B}^{D}$ becomes larger than $\Delta H_{G B}^{m}$. This means that in this situation a contribution of vacancy formation occurs. These vacancies are formed by fluctuations in the arrangement of the free space at the interface. A value of $\Delta H_{G B}^{D}$ in iron has been determined by first quantifying $\Delta H_{G B}^{m}$ by molecular dynamics simulation and then adding $\Delta H_{G B}^{f}$ (cf. equation (9)), determined from the energy change of the system after removing an atom at the boundary[19]. Determination in a similar way of a value of $\Delta H_{G B}^{f}$ for system $\mathrm{A}$ in the present work provides a value of $1.6 \mathrm{eV}$ (unrelaxed vacancy formation). This leads to a value of $\sim 3.0 \mathrm{eV}$ for $\Delta H_{G B}^{D}$, which is much higher than any of the $\Delta H_{G B}^{D}$ values determined directly from the simulations (during the transformation). Clearly, two different interpretations of what constitutes $\Delta H_{G B}^{D}$ are at stake here. As shown here, vacancy formation by thermal fluctuations in the distribution of the excess volume has a lower formation enthalpy than as found by calculations where one atom is removed from the boundary (and placed on the surface). Evidently, the latter, classical concept [19] provides only a good description of vacancy formation in interfaces if coherent interfaces are dealt with where the amount of excess volume is minimal. For incoherent interface the vacancy formation enthalpy should be determined from dynamics simulations.

As discussed in section 3.2, $\Delta H_{G B}^{D}$ increases when the amount of excess volume is decreased by adding 2 atoms to the system, but when 2 more atoms (for a total of 4 added atoms) are added $\Delta H_{G B}^{D}$ decreases again. The diffusion rate, however, decreases continuously when more and more atoms are added to the system. In the systems with 0 and with 4 added atoms $\Delta H_{G B}^{D} \approx \Delta H_{G B}^{m}$ which means that the vacancy formation enthalpy is approximately zero. Because the diffusion rate is lower for the system with 4 added atoms the number of mobile vacancies must be much smaller than in the system with 0 added atoms. In the system with 2 added atoms there are more mobile vacancies (as compared to the system with 4 added atoms) but these are more difficult to form, leading to the higher value for $\Delta H_{G B}^{D}$. There is no single value for the formation enthalpy of a vacancy at an interface.

The influence of the extra added atoms is larger for the boundary diffusion than for the interface mobility (see sections 3.1 and 4.1). Apparently, vacancy formation is more sensitive to the total amount of free volume available than the continuous fluctuations in the free volume rearrangements required for the transformation from $\gamma$ to $\alpha$. 
As a results of the analysis presented in this work it follows that a general validation of both equation (6) (boundary vs. volume diffusion) and equation (7) (interface mobility vs. volume diffusion) cannot be given: the amount of excess volume, and thus the type of interface/boundary and its orientation, determine the quantative relations of $\Delta H^{a}, \Delta H_{G B}^{D}$ and $\Delta H_{V}^{D}$. Yet, it follows from figures 6 and 7 unambiguously

$$
\Delta H^{a}<\Delta H_{G B}^{D}
$$

different from the classical estimate $\left(\Delta H^{a} \approx \Delta H_{G B}^{D}\right)$ as in Refs. [8,10] where grain growth was simulated with molecular dynamics (see also section 1). The contradiction with experimental findings (indicating $\Delta H^{a}>\Delta H_{G B}^{D}$; equations (6) and (7)) can probably be attributed to the presence of impurities in real experiments which are absent in the computer simulations.

\section{Conclusion}

Not only the amount of excess volume associated with an interface boundary but also its distribution along the interface (which depends on the crystallography of the interface) control the activation enthalpies of the interface mobility and of the boundary self diffusion. More excess volume leads to a higher interface mobility and faster diffusion. The interface mobility is higher when more free volume is available at the interface because it is easier for the atoms to jump from one crystal to the other. The diffusion is faster with more excess volume because the vacancy formation enthalpy is lower with more excess volume. During the phase transformation vacancies are formed at the interface by fluctuations in the distribution of the excess volume at the interface. The formation enthalpy of these vacancies is a distribution of different values, some vacancies are easily formed whereas others cost more energy. The effect of an increase in excess volume is stronger for diffusion than for interface mobility.

An assessment of the formation energy of vacancies at an incoherent interface should be obtained from dynamics simulations.

The interface mobility activation enthalpy $\left(\Delta H^{a}\right)$ is generally slightly lower than the bulk vacancy diffusion migration enthalpy $\left(\Delta H_{V}^{m}\right)$. The boundary vacancy diffusion migration enthalpy $\left(\Delta H_{G B}^{m}\right.$ ) is slightly larger than the bulk vacancy diffusion migration enthalpy $\left(\Delta H_{V}^{m}\right)$, because vacancy jumps at the interface can be energetically unfavourable, whereas vacancy jumps in the bulk do not change the system energy.

The activation enthalpy for the interface mobility as determined from the simulations is smaller than the activation enthalpy for boundary self diffusion $\left(\Delta H_{G B}^{D}\right.$ ). This is opposite to the usual experimental findings, which is ascribed to the absence of impurities in the simulations. 


\section{References}

[1] J. W. Christian, The theory of transformations in metals and alloys (Pergamon Press, Oxford, 2002).

[2] C. Bos, F. Sommer and E. J. Mittemeijer, Acta. Mater. 535333 (2005).

[3] P. Hänggi, P. Talkner and M. Borkovec, Rev. Mod. Phys. 62251 (1990).

[4] C. Bos, F. Sommer and E. J. Mittemeijer, Acta. Mater. 523545 (2004).

[5] C. Bos, F. Sommer and E. J. Mittemeijer, Modelling Simul. Mater. Sci. Eng. 14 273 (2006).

[6] P. J. Shewmon, Diffusion in solids, 2nd edition (TMS publication, 1989).

[7] N. F. Mott, Proc. Phys. Soc. 60391 (1948).

[8] B. Schönfelder, G. Gottstein and L. S. Shvindlerman, Acta. Mater. 531597 (2005).

[9] H. Zhang, M. Upmanyu and D. J. Srolovitz, Acta. Mater. 5379 (2005).

[10] H. Zhang and D. J. Srolovitz, Acta. Mater. 54623 (2006).

[11] M. Kaukonen, J. Perajoki, R. M. Nieminen, G. Jungnickel and Th. Frauenheim. Phys. Rev. B 61980 (2000).

[12] R. A. Johnson and D. J. Oh, J. Mater. Res. 41195 (1989).

[13] D. Frenkel and B. Smit, Understanding Molecular Simulation (Academic Press, San Diego, 1996).

[14] V. N. Perevezentsev, Phys. Met. Metallogr. 83207 (2002).

[15] J. Mandel, The statistical analysis of experimental data (Interscience publishers, 1964).

[16] H. van Swygenhoven and P. M. Derlet, Phys. Rev. B 64224105 (2001).

[17] K. Nadarzinski and F. Ernst, Phil. Mag. A 74641 (1996).

[18] H. van Swygenhoven and A. Caro, Appl. Phys. Lett. 711652 (1997).

[19] R. W. Balluffi, T. Kwok, P. D. Bristowe, A. Brokman, P. S. Ho and S Yip, Scripta Met. 15951 (1981). 


\section{Figure captions}

Figure 1. The energy of the system during the process of an atom at the interface detaching from one crystal and attaching to the other crystal.

Figure 2. The change in system energy caused by an atomic jump between positions $\mathrm{A}$ and $\mathrm{B}, \Delta U$, is shown together with the change in system energy during the jump giving the energy barrier part, $Q$, of the activation energy.

Figure 3. A possible variation in system energy on a path from the $\gamma$ phase to the $\alpha$ phase (right to left) with two intermediate states. The overall activation energy for this process $\Delta \mathrm{H}^{\mathrm{a}}$ is the difference between the maximum energy level and the energy level of the begin state.

Figure 4. A close-up of a small fragment of the interface between $\gamma$ (fcc, light grey) and $\alpha$ (bcc, dark grey) (snapshot taken during a (simulated) $\gamma \rightarrow \alpha$ transformation). The big spheres indicate occupied lattice sites, the small spheres are unoccupied lattice sites. The dashed lines show that many empty bcc sites cannot be occupied because a too strong overlap with neighbouring fcc atoms would occur.

Figure 5. A typical start configuration for the simulations. The extra space between the planes is not real; it has been inserted to provide a clearer view. Fcc atoms are light grey, bcc atoms are dark.

Figure 6. The interface mobility activation energy for system A as a function of the random site density in the system, in the absence of added atoms (i.e. system $\left.\mathrm{A}_{2}\right)$ and for 2 added atoms $\left(N_{\text {ran }}=0,4,8\right.$ and $12)$ and for 4 added atoms $\left(N_{\text {ran }}=4\right.$ and 8$)$.

Figure 7. The boundary self diffusion activation energies for system $\mathrm{A}_{2}$ as a function the random site density in the system. See section 3.2 for a description of the different experiments.

Figure 8. Mismatch patterns at the interfaces of the four systems from Table . Fcc sites are coloured light and bcc sites are coloured dark. (a) System A, (b) System B, (c) System C and (d) System D.

Figure 9. Close up of the mismatch pattern of system D (Figure 8(d)) showing, as enclosed by ellipses, the (nearly) coherent regions.

\section{Table captions}

Table 1. The different systems. All systems have fcc(111)//bcc(110). The table shows the parallel directions (in fcc and bcc). $N_{\text {plane }}$ is the number of atoms per plane parallel to the interface. The twist angle $\theta$ is defined here as zero for system $\mathrm{A}$.

Table 2. Boundary self diffusion activation enthalpy for system $A_{2}$ with $\mathrm{N}_{\text {ran }}=12$. The standard error as obtained from the fit procedure [15] is denoted by $\sigma$.

Table 3. Interface mobility activation enthalpy with $N_{\text {ran }}=0$ for the four different systems (see table 1). The standard error as obtained from the fit procedure [15] is denoted by $\sigma$. All systems have the same number of missing atoms per interface line length.

Table 4. Interface mobility activation enthalpy with Nran $=4$ for the four different systems (see table 1). The standard error as obtained from the fit procedure [15] is denoted by $\sigma$. All systems have the same number of missing atoms per interface line length.

Table 5, interface energy and percentage of overlapping fcc and bcc sites (see section 3.3) for the four different systems (see table 1). 
Figure 1

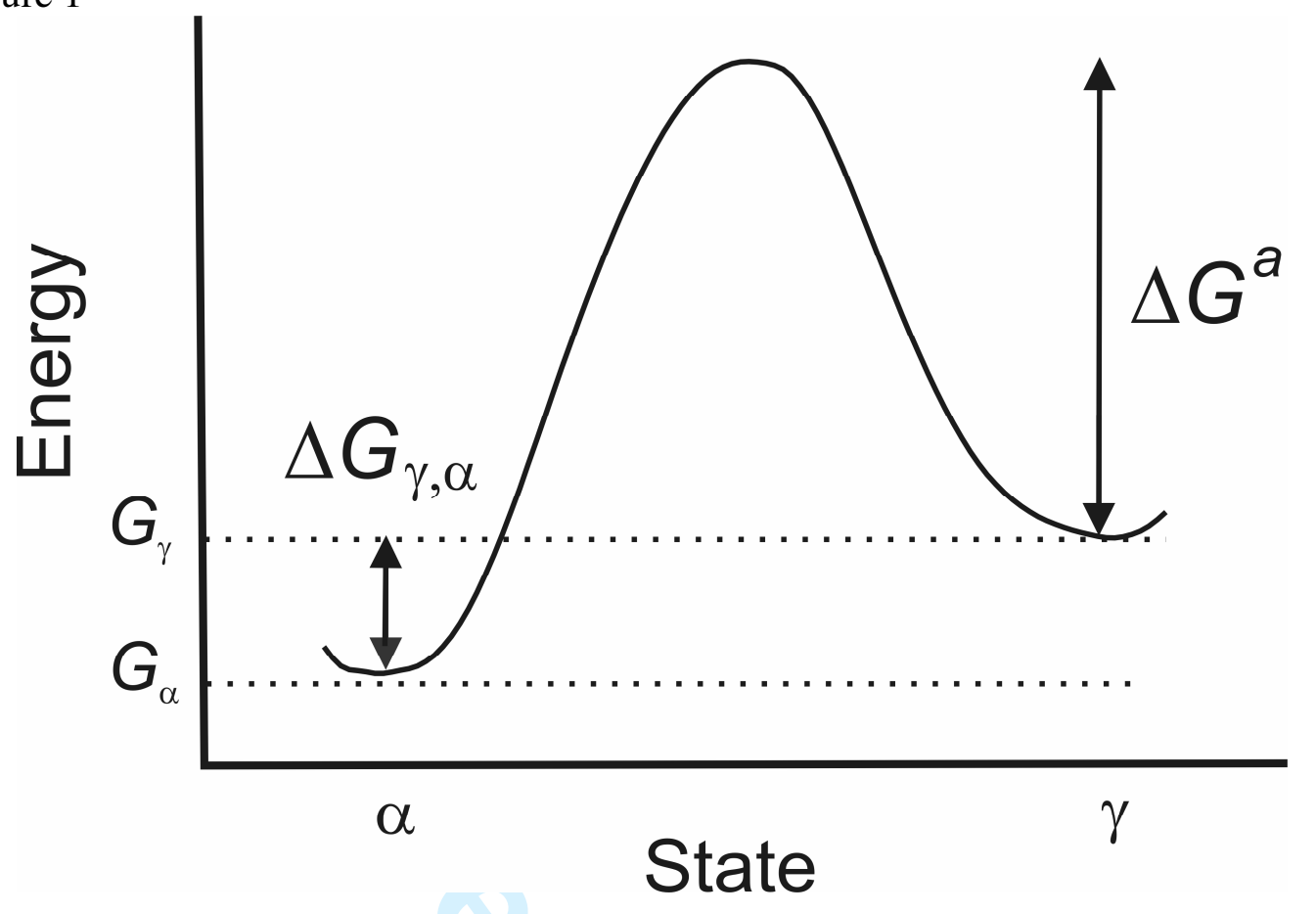

State 
Figure 2

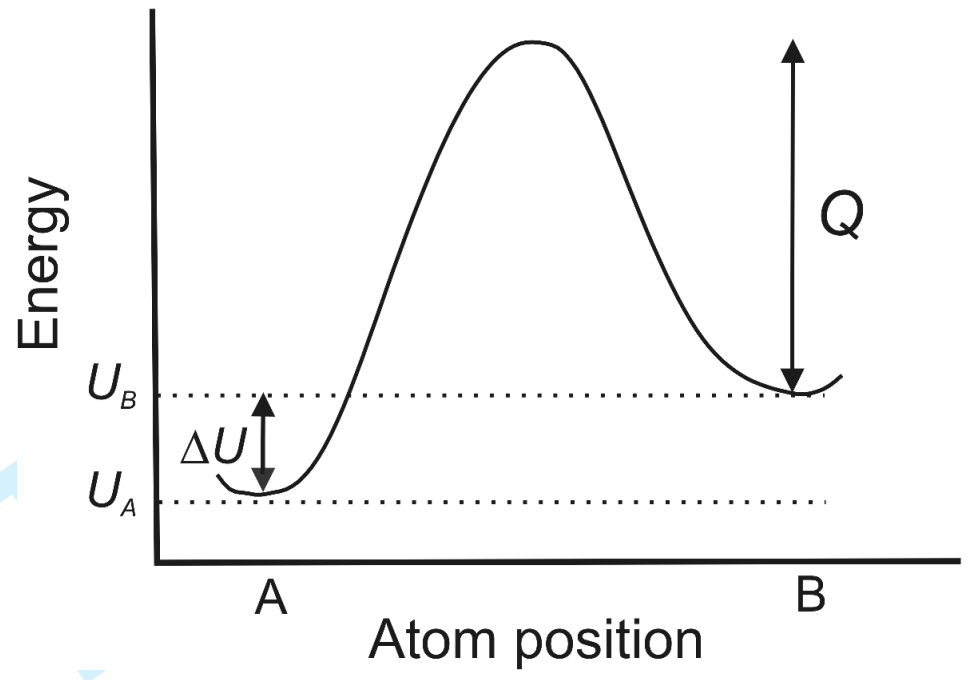


Figure 3

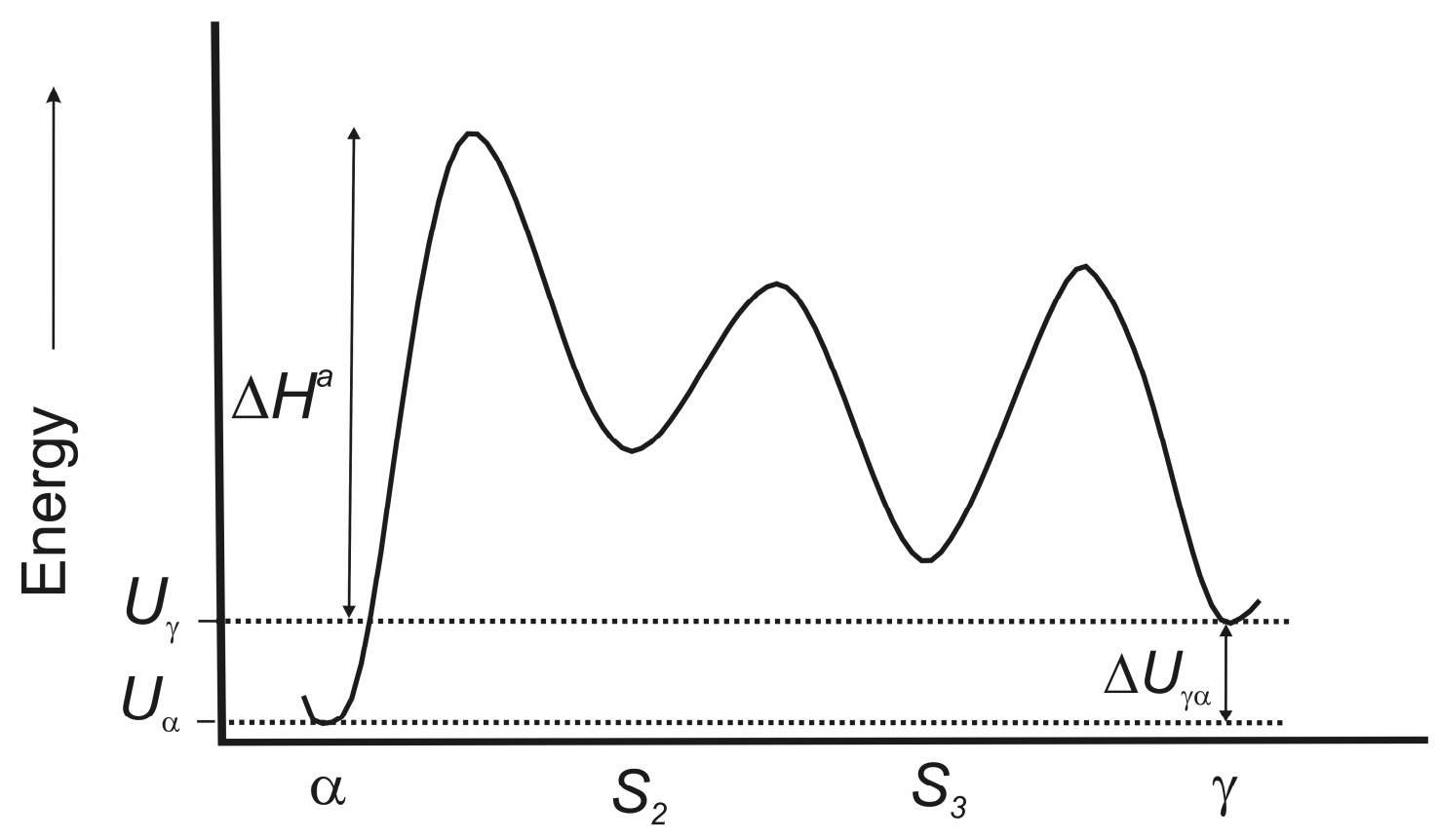

State 
Figure 4

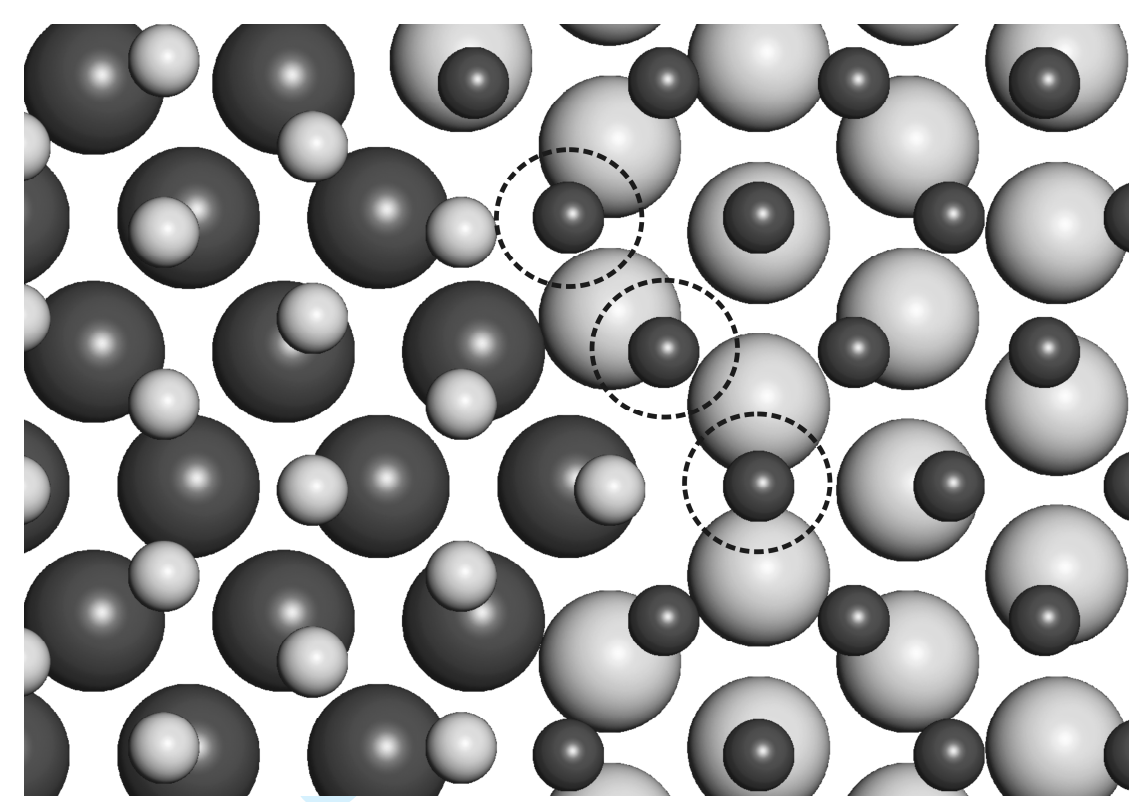


Figure 5

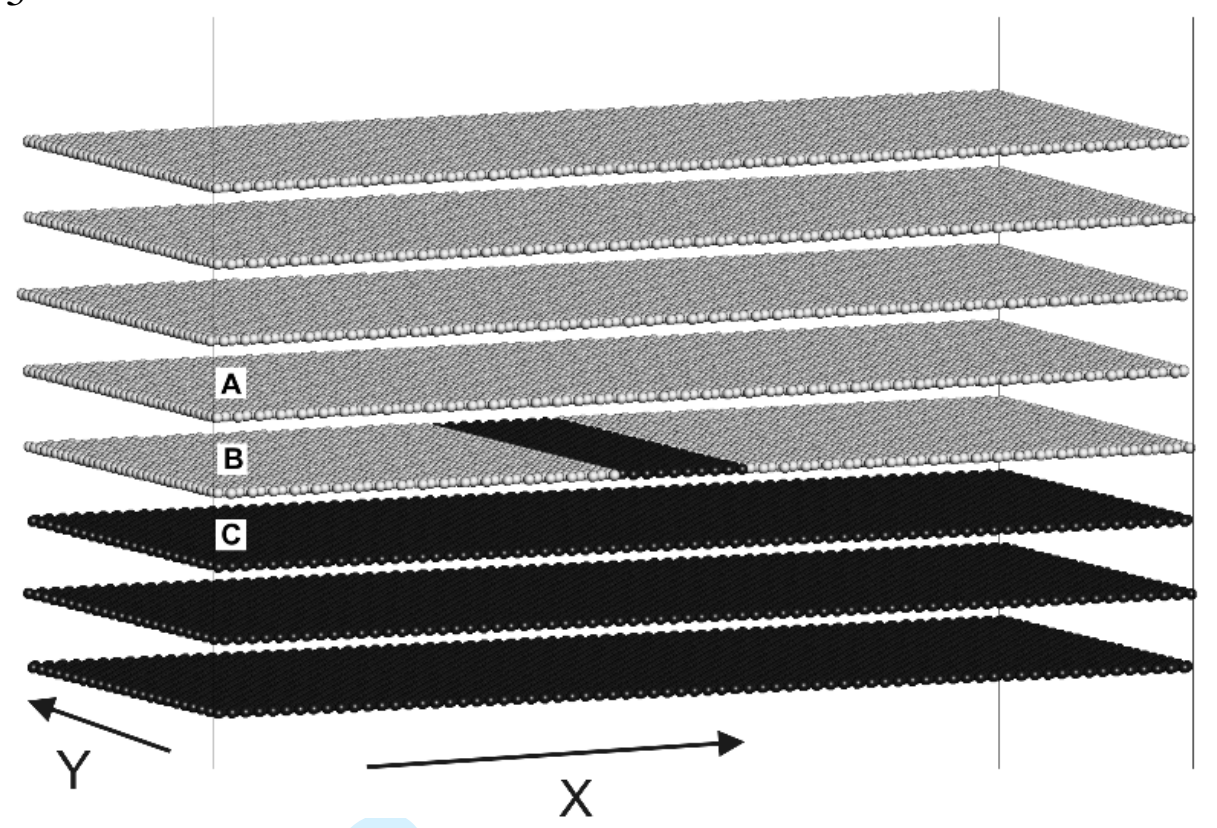


Figure 6

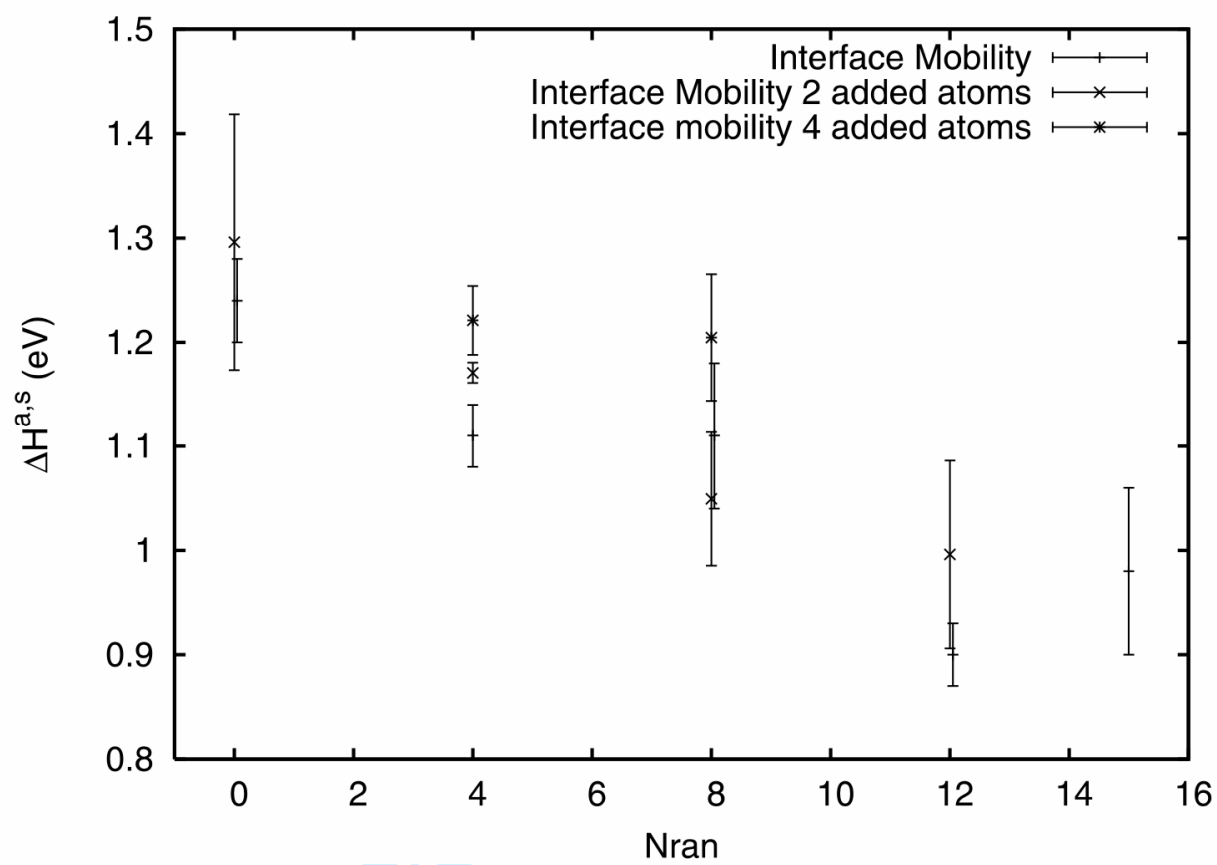


$\begin{array}{ll}1 & \\ 2 & \\ 3 & \text { Figure } 7 \\ 4 & \end{array}$

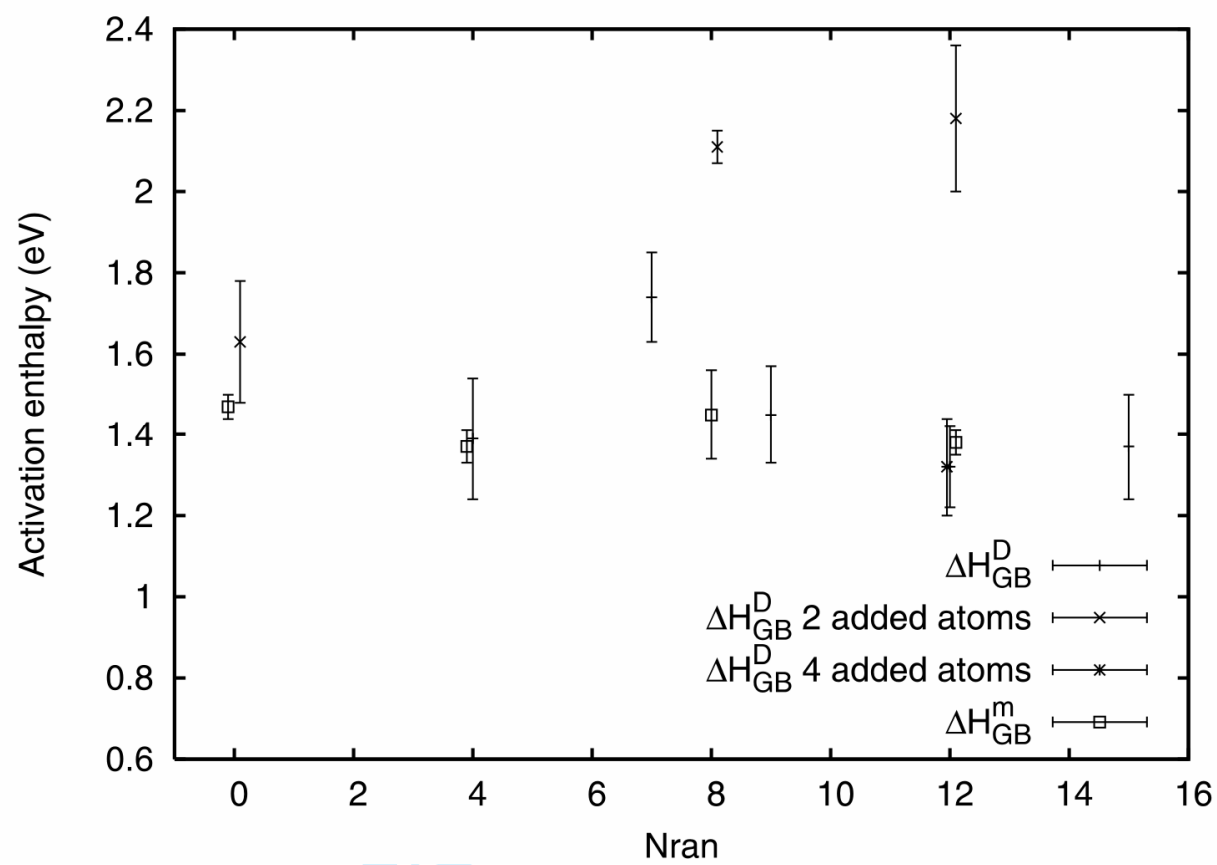


Figure 8

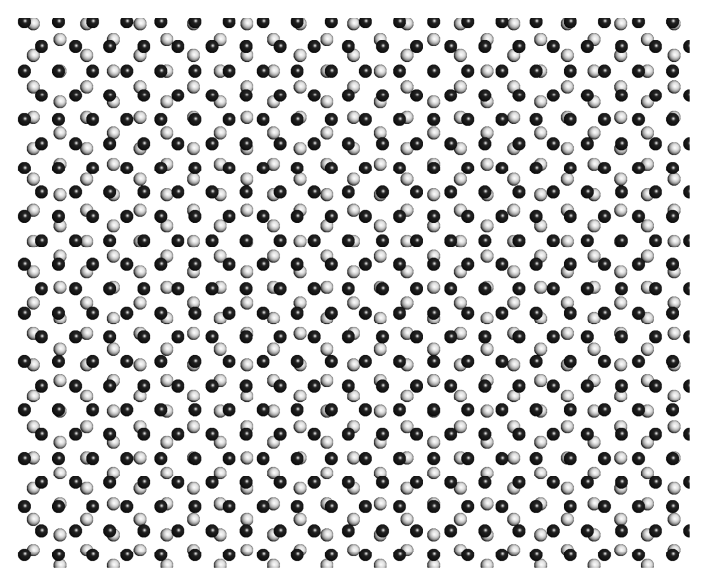

(a)

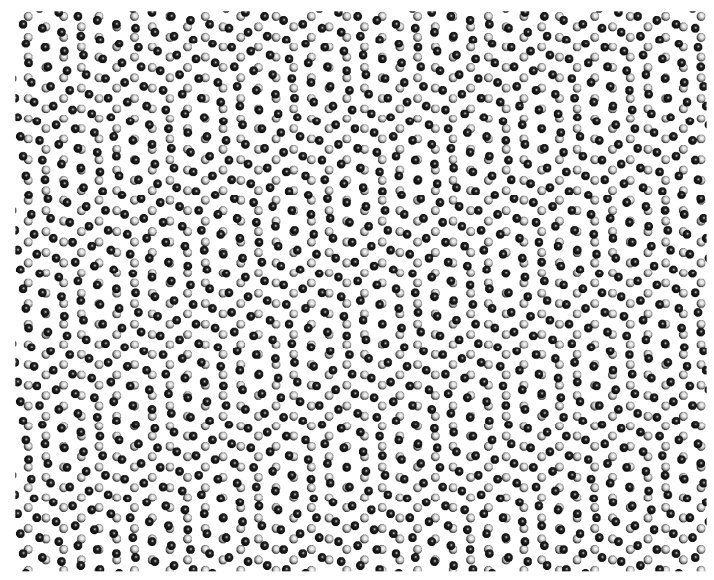

(c)

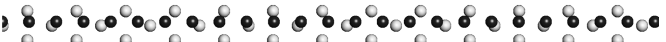

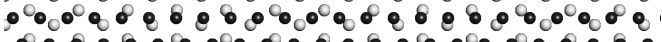

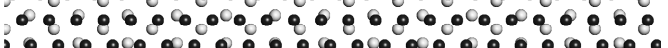

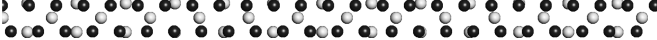

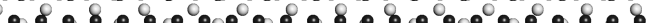

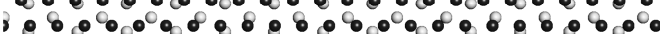

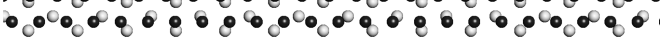
.08 .80 .000 .808000000 .800 $1: 0.000000 .: 0.000000 .0 .0100$ - $00^{\circ} 0.8080 .0000 .8080 .0000$

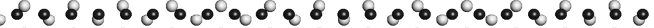

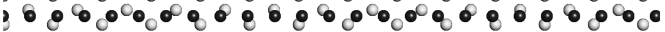

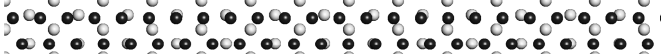

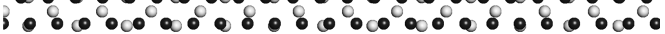

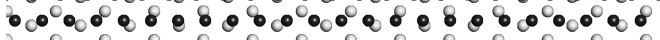
8.8 .8 .0 .0 .8 .8 .8 .0 .000 .8 .8

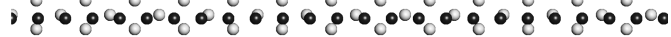
00000.000000000 .00000000

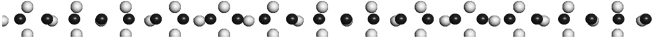

(b)

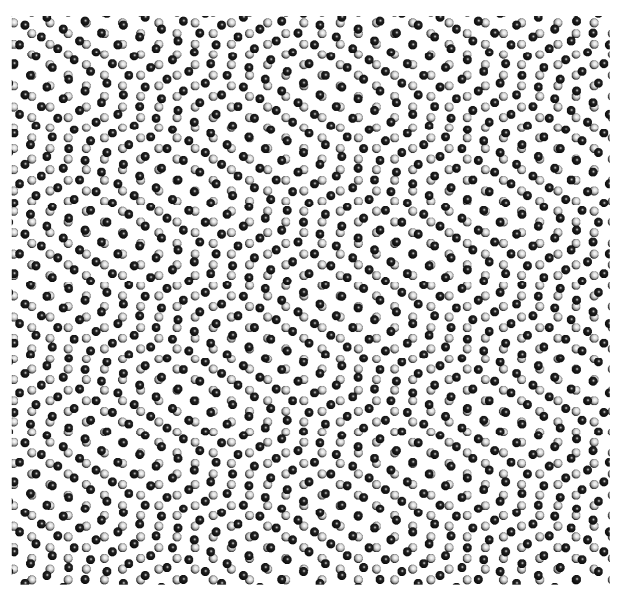

(d)

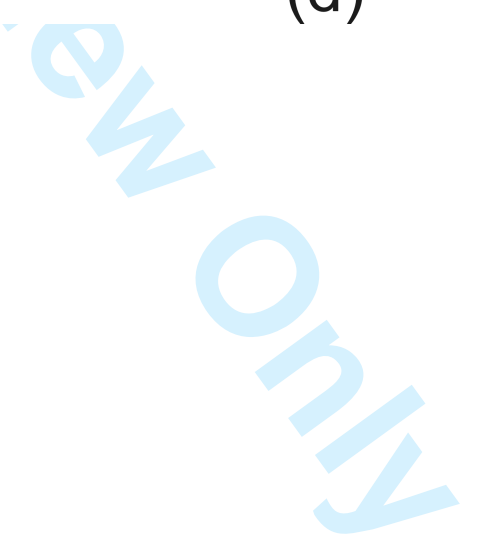


Figure 9

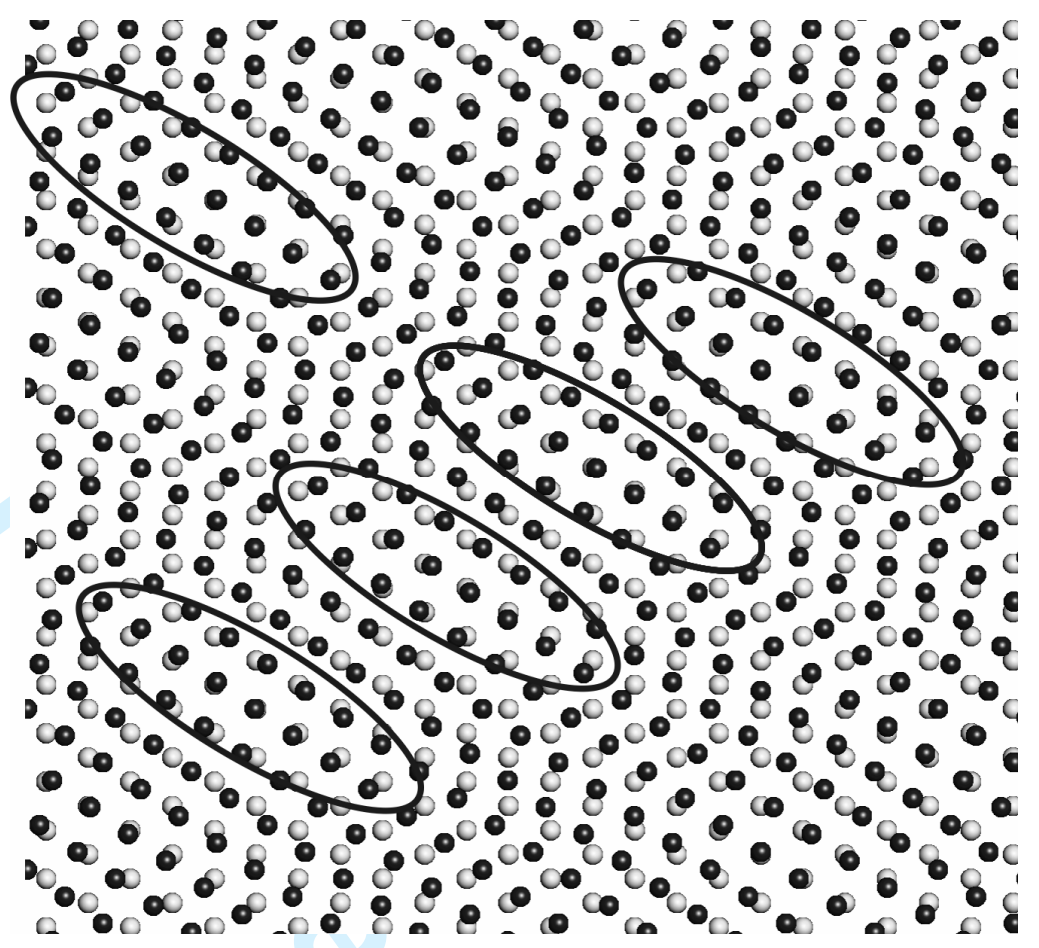


Table 1. The different systems. All systems have fcc(111)//bcc(110). The table shows the parallel directions (in fcc and bcc). $N_{\text {plane }}$ is the number of atoms per plane parallel to the interface. The twist angle $\theta$ is defined here as zero for system $A$.

\begin{tabular}{ccccc}
\hline Name & Bcc direction & Fcc direction & $N_{\text {plane }}$ & $\theta\left(^{\circ}\right)$ \\
\hline $\mathrm{A} / \mathrm{A}_{2}$ & {$[00 \overline{1}]$} & {$[11 \overline{2}]$} & 3312 & 0 \\
$\mathrm{~B}$ & {$[\overline{1} 11]$} & {$[11 \overline{2}]$} & 12690 & 54.7 \\
$\mathrm{C}$ & {$[\overline{2} 23]$} & {$[11 \overline{2}]$} & 30672 & 43.3 \\
$\mathrm{D}$ & {$[\overline{1} 14]$} & {$[11 \overline{2}]$} & 27888 & 19.5 \\
\hline
\end{tabular}


Table 2. Boundary self diffusion activation enthalpy for system $\mathrm{A}_{2}$ with $N_{\text {ran }}=12$. The standard error as obtained from the fit procedure [15] is denoted by $\sigma$.

\begin{tabular}{ccccc}
\hline Added atoms & $\begin{array}{c}\Delta H_{G B}^{D} \\
{[\mathrm{eV}]}\end{array}$ & $\begin{array}{c}\sigma_{\Delta H_{G B}^{D}} \\
{[\mathrm{eV}]}\end{array}$ & $\begin{array}{c}C^{\prime \prime} \\
{\left[10^{-21} \mathrm{~m}^{2} \mathrm{~s}^{-1}\right]}\end{array}$ & $\begin{array}{c}\sigma_{C^{\prime \prime}} \\
{\left[10^{-21} \mathrm{~m}^{2} \mathrm{~s}^{-1}\right]}\end{array}$ \\
\hline 0 & 1.32 & 0.1 & 0.5 & 0.2 \\
2 & 2.18 & 0.18 & 9 & 7 \\
4 & 1.32 & 0.12 & 0.13 & 0.06 \\
\hline
\end{tabular}


Table 3. Interface mobility activation enthalpy with $N_{\text {ran }}=0$ for the four different systems (see table 1). The standard error as obtained from the fit procedure [15] is denoted by $\sigma$. All systems have the same number of missing atoms per interface line length.

\begin{tabular}{ccccc}
\hline Name & $\begin{array}{c}\Delta H^{a, s} \\
{[\mathrm{eV}]}\end{array}$ & $\begin{array}{c}\sigma_{\Delta H a, s} \\
{[\mathrm{eV}]}\end{array}$ & $\begin{array}{c}C \\
{\left[10^{15} \text { atoms/sec }\right]}\end{array}$ & $\begin{array}{c}\sigma_{C} \\
{\left[10^{15}\right.}\end{array}$ atoms/sec $]$ \\
\hline $\mathrm{A}$ & 1.08 & 0.13 & 0.6 & 0.4 \\
$\mathrm{~B}$ & 1.11 & 0.03 & 1.2 & 0.1 \\
$\mathrm{C}$ & 1.36 & 0.08 & 3.9 & 1.2 \\
$\mathrm{D}$ & 1.41 & 0.07 & 5.3 & 1.6 \\
\hline
\end{tabular}


Table 4 . Interface mobility activation enthalpy with $N_{\text {ran }}=4$ for the four different systems (see table 1 ). The standard error as obtained from the fit procedure [15] is denoted by $\sigma$. All systems have the same number of missing atoms per interface line length.

\begin{tabular}{ccccc}
\hline Name & $\begin{array}{c}\Delta H^{a, s} \\
{[\mathrm{eV}]}\end{array}$ & $\begin{array}{c}\sigma_{\Delta H a, s} \\
{[\mathrm{eV}]}\end{array}$ & $\begin{array}{c}C \\
{\left[10^{15} \text { atoms/sec }\right]}\end{array}$ & $\begin{array}{c}\sigma_{C} \\
{\left[10^{15} \text { atoms/sec }\right]}\end{array}$ \\
\hline $\mathrm{A}$ & 1.14 & 0.06 & 1.3 & 0.4 \\
$\mathrm{~B}$ & 1.04 & 0.07 & 0.6 & 0.2 \\
$\mathrm{C}$ & 1.29 & 0.02 & 2.0 & 0.1 \\
$\mathrm{D}$ & 1.65 & 0.12 & 10.6 & 5.3 \\
\hline
\end{tabular}


Table 5, interface energy and percentage of overlapping fcc and bcc sites for the four different systems (see table 1).

\begin{tabular}{ccc}
\hline System Name & $\begin{array}{c}\text { Overlap } \\
{[\%]}\end{array}$ & $\begin{array}{c}\gamma \\
{\left[\mathrm{Jm}^{-2}\right]}\end{array}$ \\
\hline A & 14.7 & 0.658 \\
B & 16.0 & 0.651 \\
C & 20.4 & 0.638 \\
D & 23.3 & 0.582 \\
\hline
\end{tabular}

